\title{
Beyond Feedforward Models Trained by Backpropagation: a Practical Training Tool for a More Efficient Universal Approximator
}

\author{
Roman Ilin*, Robert Kozma†, Paul J. Werbos ${ }^{\frac{\Phi}{}}$
}

October 22, 2018

Paper to appear in IEEE Transactions on Neural Networks

\begin{abstract}
Cellular Simultaneous Recurrent Neural Network (SRN) has been shown to be a function approximator more powerful than the MLP. This means that the complexity of MLP would be prohibitively large for some problems while SRN could realize the desired mapping with acceptable computational constraints. The speed of training of complex recurrent networks is crucial to their successful application. Present work improves the previous results by training the network with extended Kalman filter (EKF). We implemented a generic Cellular SRN and applied it for solving two challenging problems: 2D maze navigation and a subset of the connectedness problem. The speed of convergence has been improved by several orders of magnitude in comparison with the earlier results in the case of maze nav-

*Roman Ilin is with Department of Computer Science at The University of Memphis, Memphis, TN 38117. E-mail: rilin@memphis.edu

${ }^{\dagger}$ Robert Kozma is with Department of Computer Science at The University of Memphis, Memphis, TN 38117. E-mail: rkozma@memphis.edu

${ }^{\ddagger}$ Paul J. Werbos, Room 675, National Science Foundation, Arlington, VA 22230. E-mail: pwerbos@nsf.gov

$\S$ The opinions expressed in this paper are of the authors and do not necessarily reflect the views of their employers, in particular NSF
\end{abstract}


igation, and superior generalization has been demonstrated in the case of connectedness. The implications of this improvements are discussed. 


\section{Introduction}

The artificial neural networks, inspired by the enormous capabilities of living brains, are one of the cornerstones of today's field of artificial intelligence. Their applicability to real world engineering problems has become unquestionable in the recent decades, see for example [1]. Yet most of the networks used in the real world applications use the feedforward architecture, which is a far cry from the massively recurrent architecture of the biological brains. The widespread use of feed-forward architecture is facilitated by the availability of numerous efficient training methods. However, the introduction of recurrent elements makes training more difficult and even impractical for most non-trivial cases.

The SRN's have been shown to be more powerful function approximators by several researchers ( $[2],[3])$. It has been shown experimentally that an arbitrary function generated by a MLP can always be learned by an SRN. However the opposite was not true, as not all functions given by a SRN could be learned by a MLP. These results support the idea that the recurrent networks are essential in harnessing the power of brain-like computing.

It is well known that MLPs and a variety of kernel-based networks (like RBF) are universal function approximators, in some sense. Andrew Barron [4] proved that MLPs are better than linear basis function systems like Taylor series in approximating smooth functions; more precisely, as the number of inputs $\mathrm{N}$ to a learning system grows, the required complexity for an MLP only grows as $O(N)$, while the complexity for a linear basis function approximator grows exponentially, for a given degree of accuracy in approximation. However, when the function to be approximated does not live up to the usual concept of smoothness, or when the number of inputs becomes even larger than what an MLP can readily handle, it becomes ever more important to use a more general class of neural network.

The area of intelligent control provides examples of very difficult functions to be tackled by ANN's. Such functions arise as solutions to multistage optimization problems, given by the Bellman equation 8, The design of non-linear control systems, also known as "Adaptive Critics", presupposes the ability of the so called "Critic network" to approximate the solution of the Bellman equation. See [5] for overview of adaptive critic designs. Such problems also are classified as Approximate Dynamic Programming (ADP). A simple example of such function is the 2D maze navigation problem, considered in this contribution. See [6] 
for in depth overview of the ADP and Maze navigation problem. The applications of EKF for NN training have been developed by researchers in the field of control [7], [8], [5].

The classic challenge posed by Rosenblatt to perception theory is the recognition of topological relations [9]. Minsky and Papert [10] have shown that such problems fundamentally cannot be solved by perceptrons because of their exponential complexity. The multi-layer perceptrons are more powerful than Rosenblatt's perceptron but they are also claimed to be fundamentally limited in their ability to solve topological relation problems [11]. An example of such problem is the connectedness predicate. The task is to determine whether the input pattern is connected regardless of its shape and size.

The two problems described above pose fundamental challenges to the new types of neural networks, just like the XOR problem posed a fundamental challenge to the perceptrons, which could be overcome only by the introduction of the hidden layer and thus effectively moving to the new type of ANN.

In this contribution, we present the Cellular Simultaneous Neural Network (CSRN) architecture. This is a case of more generic architecture called ObjectNet, see [12], chapter 6, page 120. We use the Extended Kalman Filter (EKF) methodology for training our networks and obtain very encouraging results. For the first time an efficient training methodology is applied to the complex recurrent network architecture. Extending the preliminary result introduced in [13], the present study addresses not only learning but also generalization of the network on two problem: maze and connectedness. Improvement in speed of learning by several orders of magnitude as a result of using EKF is also demonstrated. We consider the results introduced in this work as initial demonstration of the proposed learning principle, which should be thoroughly studied and implemented in various domains.

The rest of this paper is organized as follows. Section 2 describes the calculation of derivatives in the recurrent network. Section 3 describes the CSRN architecture. Section 4 gives the EKF formulas. Section 5 describes the operation of a generic CSRN application. Sections 6 and 7 describe the two problems addressed by this contribution and give the simulation results. Section 8 is the discussion and conclusions. 


\section{Backpropagation in complex networks}

The backpropagation algorithm is the foundation of neural network applications [14], [15]. Backpropagation relies on the ability to calculate the exact derivatives of the network outputs with respect to all the network parameters.

Real live applications often demand complex networks with large number of parameters. In such cases, the use of the rule of ordered derivatives [14], [16] allows to obtain the derivatives in systematic manner. This rule also allows to simplify the calculations by breaking a complex network into simple building blocks, each characterized by its inputs, outputs and parameters. If the derivatives of the outputs of a simple building block with respect to all its internal parameters and inputs are known, then the derivatives of the complete system can be easily obtained by backpropagating through each block.

Suppose that the network consists of N units, or subnetworks, which are updated in order from 1 to $\mathrm{N}$. We would like to know the derivatives of the network outputs with respect to the parameters of each unit. In general case the final calculation for any network output $j$ is a simple summation:

$$
\frac{\partial^{+} z_{j}}{\partial \alpha}=\sum_{i=1}^{N} \sum_{k=1}^{n} \delta_{k}^{i} \frac{\partial^{+} z_{k}^{i}}{\partial \alpha}
$$

here $\alpha$ stands for any parameter, $i$ is the unit number, $k$ is the index of the output of the current unit, $\delta_{k}^{j}$ is the derivative with respect to the input of the unit that is connected to the $k^{\text {th }}$ output of the $i^{\text {th }}$ unit. Note that the $k^{\text {th }}$ output of the current unit can feed into several subsequent units and so the "delta" will be a sum of the "deltas" obtained from each unit. Also $\delta_{k}^{N}$ 's are set externally as if the network were a part of a bigger system. If we simply want the derivatives of the outputs, set $\delta_{k}^{N}=1$. We provide an example of this calculation in Appendix A.

Let's denote the outputs of our network as $z_{i}$. Ultimately we are interested in obtaining the derivatives of these outputs w.r.t. all the internal parameters. This is equivalent to calculating the Jacobian matrix of the system. For example, if we have two outputs and three internal parameters a,b, and c, the Jacobian will be

$$
\bar{C}=\left(\begin{array}{ccc}
\frac{\partial^{+} z_{1}}{\partial a} & \frac{\partial^{+} z_{1}}{\partial b} & \frac{\partial^{+} z_{1}}{\partial c} \\
\frac{\partial^{+} z_{2}}{\partial a} & \frac{\partial^{+} z_{2}}{\partial b} & \frac{\partial^{+} z_{2}}{\partial c}
\end{array}\right)
$$


This matrix can be used to adjust the system's parameters using various methods such as gradient descent or Extended Kalman Filter.

So far we considered multi-layered feed-forward networks. The methodology described above can be extended to recurrent networks. Let us consider a feed-forward network with recurrent connections that link some of its outputs to some of its inputs. Suppose that the network is updated for $\mathrm{N}$ steps. We would like to calculate the derivatives of the final network outputs w.r.t. the weights of the network. This calculation is a case of Eq. 1. Suppose that the network has $m$ inputs, and $n$ outputs. We assume that the expressions for the derivatives of all outputs w.r.t. each input and each network weight $\partial z_{k} / \partial \alpha, k=1 . . n$, $\partial z_{k} / \partial x_{p}, k=1 . . n, p=1 . . m$ are known and we will also denote the ordered derivatives $\partial^{+} z_{k} / \partial \alpha$ by $F_{\alpha}^{k}$. Then the full derivatives calculation is given by the algorithm in Fig. 1. Note that we omit the loop over all the weight parameters $\alpha$ to improve readability. The result of this algorithm is the Jacobian matrix of the network after $\mathrm{N}$ iterations.

\section{Cellular Simultaneous Recurrent Networks}

SRN's can be used for static functional mapping, similarly to the MLP's. They differ from more widely known time lagged recurrent networks (TLRN) because the input in SRN is applied over many time steps and the output is read after the initial transitions have disappeared and the network is in equilibrium state. The most critical difference between TLRN and SRN is whether the network output is required at the same time step (TLRN) or after the network settles to an equilibrium (SRN).

Many real live problems require to process patterns that form a $2 \mathrm{D}$ grid. For instance, such problems arise in image processing or in playing a game of chess. In those cases the structure of the neural network should also become a $2 \mathrm{D}$ grid. If we make all the elements of the grid identical, the resulting cellular neural network benefits from greatly reduced number of independent parameters.

The combination of cellular structure with SRN creates very powerful function approximators. We developed a CSRN package that can be easily adopted to various problems. The architecture of the network is given in Fig. 2. The input is always a $2 \mathrm{D}$ grid. The number of cells in the network equals to the size of the input. The number of outputs also equals to the size of the input. Since most of the problems require only few outputs, we 
add an arbitrary output transformation to the network. It has to be differentiable, but it does not have to have adjustable parameters. The training in our applications occurs only in the SRN. The cells of the network are connected through neighbor links. Each cell has four neighbors and the edges of the network wrap around.

The cell of CSRN in our implementation is a generalized MLP [17], shown in Fig. 3. Each non-input node of the GMLP is linked to all the subsequent nodes thus generalizing the idea of multi-layered network. The recurrent connections come from the output nodes of this cell and from its neighboring cells. It is an important feature of the used architecture that each cell has the same weights, which allows to build arbitrary large networks without increasing the number of weight parameters.

\section{Extended Kalman Filter for Network Training}

Kalman filters (KF) originated in signal processing. They present a computational technique which allows to estimate the hidden state of a system based on observable measurements. See [18], [19] for derivation of the classical Kalman filter formulas based on the theory of multivariate normal distribution.

In the case of neural network training, we are faced with the problem of determining the parameter weights in such a way that the measured outputs of the network are as close to the target values as possible. The network can be described as a dynamical system with its hidden state vector $\vec{W}$ formed by all the values of network weights, and the observable measurements vector formed by the values of network outputs $\vec{Y}$. It is sometimes convenient to form a full state vector $\vec{S}$ that consists of both hidden and observable parts. Such formulation can be used in the derivation of Kalman filter [18]. In this paper we follow the convention in the literature which refer to $\vec{W}$ as the state vector [20]. Note that the outputs of the network can be expressed in terms of the weights as

$$
\vec{Y}=\bar{C} \vec{W}
$$

where $\bar{C}$ is the Jacobian matrix of the network evaluated around the current output vector $\vec{Y}$. We assume that the state $\vec{S}$ is normally distributed and we are interested in the estimate of $\vec{W}$ based on the knowledge of $\vec{Y}$ and the underlying dynamical model of the system, which is simply $\vec{Y}(i+1)=\vec{t}$ and $\vec{W}(i+1)=\vec{W}(i)$, where $\vec{t}$ is the target output 
of the network. Suppose the covariance matrix of $\vec{W}$ is given by $\bar{K}$, and the measurement noise covariance matrix by $\bar{R}$. $\bar{R}$ is assumed to be normally distributed with zero mean. Then the Kalman update is given by the following formulas.

$$
\begin{gathered}
\vec{W}(i+1)=\vec{W}(i)+\frac{\bar{K}(i) \bar{C}(i)^{T}}{\bar{C}(i) \bar{K}(i) \bar{C}(i)^{T}+\bar{R}(i)}(\vec{t}-\vec{Y}(i)) \\
\bar{K}(i+1)=\bar{K}(i)-\frac{\bar{K}(i) \bar{C}(i)^{T} \bar{C}(i) \bar{K}(i)}{\bar{C}(i) \bar{K}(i) \bar{C}(i)^{T}+\bar{R}(i)}+\bar{Q}(i)
\end{gathered}
$$

We introduced the index i to denote current training step. The matrix $\bar{Q}(i)$ is the process noise covariance matrix. It represents our assumptions about the distribution of the true values of $\vec{W}$. The formulas 4 and 5 are the celebrated Extended Kalman filter formulas which can be found in numerous literature, see for example [21], [20] , [8]. If we look closely at 4 , we can see the similarity between the EKF update and the regular gradient descend update. In both cases we have some matrix coefficient multiplied by the difference $(\vec{t}-\vec{Y}(i))$. In the case of gradient descend, the coefficient is simply $\bar{C}(i)$ multiplied by some learning rate. In the case of EKF, the coefficient is more complex as it involves the covariance matrix $\bar{K}$, which is the key to the efficiency of EKF.

The process noise $\bar{Q}$ can be safely assumed to be 0 , even though setting it to a non-zero value helps prevent $\bar{K}$ from becoming negative definite and destabilizing the filter. The measurement noise $\bar{R}$ also plays an important role in fine tuning the EKF by accelerating the speed of learning. The proper functioning of EKF depends on the assumption that the state vector $\vec{S}$ is normally distributed. This assumption usually does not hold in practice. However, adding the normally distributed noise described by $\bar{R}$ helps overcome this difficulty. $\vec{R}$ is usually chosen to be a random diagonal matrix. The values on the diagonal are annealed as the network training progresses, so that by the end of training, noise is insignificant. It turned out that the way $R$ is annealed has significant effect on the rate of convergence. After experimenting with different functional forms we used the following formula:

$$
R(i)=\operatorname{alog}\left(b \vec{\delta}(i)^{2}+1\right) I
$$

where $\delta(i)^{2}$ is the squared error, $\delta(i)=\vec{t}-\vec{Y}(i)$. The constants $a$ and $b$ were determined experimentally. We used $a=b=0.001$, which gave reasonably good results presented in 
the next sections. This functional form works better than linear annealing. Making the measurement noise a function of the error results in fast and reliable learning.

The algorithm described above is suitable for learning one pattern. Learning multiple patterns creates additional challenges. The patterns can be learned one by one or in a batch. In the present work we used the batch mode which results in efficient learning but is computationally demanding. To explain this method, we write Eq. 4 more compactly as

$$
\delta \vec{W}=\bar{G} \vec{\delta}
$$

where $\bar{G}=\bar{K} \bar{C}^{T} /\left(\bar{C} \bar{K} \bar{C}^{T}+\bar{R}\right)$ and the time step index is omitted for clarity. The matrix $\bar{G}$ is called Kalman gain.

Suppose that the network has s outputs and p weights. The size of matrix $\bar{C}$ is s by $\mathrm{p}$, and the size of $\bar{G}$ is p by s. Suppose we have M patterns in a batch. If the network is duplicated $\mathrm{M}$ times, the resulting network will have $M \times s$ outputs. The size of $\bar{C}$ becomes $M \times s$ by p. Note that we simply concatenate $\mathrm{M}$ matrices together. Matrix $\bar{G}$ can still be computed from Eq. 4 and its size becomes p by $M \times s$. The weight update can be done just like in the case of one pattern, except now the matrix $\bar{G}$ encodes information about all patterns.

This method is called multi-streaming [22], [23]. Increasing number of input patterns will result in large sizes of $\bar{C}$ and $\bar{G}$. This will make batch update inefficient because of the need to invert large matrices. Therefore, larger problems will require more advanced numerical techniques already used by practitioners of EKF training [24], [22].

\section{CSRN training algorithm}

The network architecture given in Fig. 2 is very generic and with proper implementation can be easily adopted to different problems. The algorithm given in Fig. 8 describes the training of CSRN. The main loop calculates the Jacobian matrix $\bar{C}$, which is used in the Kalman weight update. We perform testing during each training period and make a decision to stop training based on testing results. Output transformation is the part of the network that has to be customized for each problem. Other network parameters include network size, cell size, number of internal steps of SRN, EKF parameters $\bar{K}, \bar{R}$, and $\bar{Q}$. The number of internal steps is selected large enough to allow the typical network to settle 
to an equilibrium state. In the process of training the network dynamics changes and sometimes it no longer settles. Currently we do not terminate training even if equilibrium is not reached as such networks still achieve good levels of generalization. The Matlab implementation is available from the authors.

\section{Application of EKF Learning to Generalized Maze Navigation Problem}

\subsection{Problem Description}

The generalized maze navigation consists of finding the optimal path from any initial position to the goal in a $2 \mathrm{D}$ grid world. An example of such a world is illustrated in Fig. 4. One version of an algorithm for solving this problem will take a representation of the maze as its input and return the length of path from each clear cell to the goal. For example, for a 5 by 5 maze the output will consist of 25 numbers. Once we know the numbers it is very easy to find the optimal path from any cell by simply following the minimum among the neighbors. Examples of such outputs are given in Fig. 5.

2D Maze Navigation is a very simple representative of a broad class of problems solved using the techniques of Dynamic Programming, which means finding the J cost-to-go function using the Bellman's equation (see for example [21]). Dynamic Programming gives the exact solution to multistage decision problems. More precisely, given a Markovian decision process with $\mathrm{N}$ possible states and the immediate expected cost of transition between any two states $i$ and $j$ denoted by $c(i, j)$, the optimal cost-to-go function for each state satisfies the following Bellman's optimality equation.

$$
J^{*}(i)=\min _{\mu}\left(c(i, \mu(i))+\gamma \sum_{j=1}^{N} p_{i j}(\mu) J^{*}(j)\right)
$$

$J(i)$ is the total expected cost from the initial state $i$, and $\gamma$ is the discount factor. The cost $J$ depends on the policy $\mu$, which is the mapping between the states and actions causing state transitions. The optimal expected cost results from the optimal policy $\mu *$. Finding such policy directly from Eq. 8 is possible using recursive techniques but computationally expensive as the number of states of the problem grows. In the case of 2D maze, the immediate cost $c(i, j)$ is always 1 , and the probabilities $p_{i j}$ can only take values of 0 or 1 . 
The $\mathrm{J}$ surface resulting from the $2 \mathrm{D}$ maze is a challenging function to be approximated by a neural network. It has been shown that an MLP cannot solve the generalized problem [2]. Therefore, this is a great problem to demonstrate the power of the Cellular SRN's. It has been shown that CSRN is capable of solving this problem by designing its weights in a certain way [25]. However the challenge is to train the network to do the same.

The Cellular SRN to solve the $\mathrm{m}$ by $\mathrm{m}$ maze problem consists of $\mathrm{m}+2$ by $\mathrm{m}+2$ grid of identical units. The extra row and column on each side result from introducing the walls around the maze which prevent the agent from running away. Each unit receives input from the corresponding cell of the maze and returns the value of the $\mathrm{J}$ function for this cell. There are two inputs for each cell, one indicates whether this is a clear cell or an obstacle and the other supplies the values of the goal. As shown in Fig. 2, the number of outputs of the cellular part of the network equals to the number of cells. In the maze application the final output is the values of $\mathrm{J}$ function for each input cell and therefore there is no need for the output transformation.

\subsection{Results of 2D Maze Navigation}

Previous results of training the Cellular SRN's showed slow convergence [2]. Those experiments used back-propagation with adaptive learning rate (ALR) [1]. The network consisted of 5 recurrent nodes in each cell and was trained on up to 6 mazes. The initial results demonstrated the ability of the network to learn the mazes [13].

The introduction of EKF significantly sped up the training of the Cellular SRN. In the case of single maze, the network reliably converges within 10-20 training cycles (see fig. 8). In comparison, back-propagation through time with adaptive learning rate (ALR) takes between 500 and 1000 training cycles and is more dependent on the initial network weights [2].

We discovered that increasing the number of recurrent nodes from 5 to 15 allows to speed up both EKF and ALR training in case of multiple mazes. Nevertheless the EKF has a clear advantage. For more realistic learning assignment we use 30 training mazes and test the network with 10 previously unseen mazes. The training targets where computed using dynamic programming algorithm. Fig. [7A shows the sum squared error as function of the training step. We can see that EKF reaches the reasonable level in 150 training cycles. 
For comparison the back-propagation through time with adaptive learning rate training is shown on the same graph.

The true solution consists of integer values with the difference of one between the neighboring cells. We suppose that the approximation is reasonable if the maximum error per cell is less than 0.5 , since in this case the correct differences will be preserved. This means that for a 7 by 7 network corresponding to 5 by 5 maze, the sum squared error has to fall below $49 * 0.5^{2}=12.25$. In Fig. $7 \mathrm{~A}$ the EKF drops below the 12.25 level within 150 steps while ALR testing saturates at the level close to 50. We use 20 internal steps within each training cycle. In practical training scenarios the error is obviously not the same for each cell. Detailed statistical analysis can reveal the true nature of the expected distributions. In the present exploratory study we don't go into the details of statistics. Rather we introduce an empirical measure of the goodness of learnt navigation task in the following way. We count how many gradients point in the correct direction. The ratio of the number of correct gradients to the total number of gradients is our goodness ratio $G$ that can vary from 0 to 100 percent. The gradient of the $\mathrm{J}$ function gives the direction of the next move. As an example, Fig. 5 shows the J function computed by a network and the true J function. Fig. 5 demonstrates 2 erroneous gradient directions. The goodness $\mathrm{G}$ is illustrated in Fig. 7B. We can see that EKF reaches testing performance of

75-80 percent after 150 training cycles averaged over 10 testing mazes. On the other hand $\mathrm{BP} / \mathrm{ALR}$ testing performance lingers around 50 percent chance level for several hundred training cycles. Even after 500 training cycles it is close to the chance level. This shows the potential of EKF for training CSRN's.

\section{Application of EKF to Connectedness Problem}

\subsection{A Simple Connectedness Problem}

The description of connectedness problem can be found in [10]. The problem consists of answering the question "is the input pattern connected?". Such question is fundamental to our ability to segment visual images into separate objects which is the first preprocessing step before trying to recognize and classify the objects. We work with a subset of the connectedness problem, where we consider a square image and ask the following question: "are 
the top left and the bottom right corners connected?". Note that the diagonal connections do not count in our case, each pixel of a connected pattern has to have a neighbor on the left, right, top or bottom. Examples of such images are given in Fig. 6. This subset is still a difficult problem which could not be solved by the feedforward network [26]. The reason why connectedness is a difficult problem lies it its sequential nature. Human eye has to follow the borders of an image sequentially in order to classify it. This explains the need for recursion.

The network architecture for the connectedness problem is that of Fig. 2, The output transformation is a GMLP with one output. The weights of this GMLP are randomly generated and fixed. The target outputs are +0.5 for connected pattern and -0.5 for disconnected pattern.

\subsection{Results of Connectedness Problem}

Here we present the results of solving the subset of connectedness problem. We applied the network to image sizes 5, 6, and 7 . In each case we generated sets of 30 random connected and 30 disconnected patterns for training, and 10 connected and 10 disconnected patterns for testing. We used 20 internal iterations and the training took between 100 and 200 training cycles. We used the same EKF parameters as in the case of maze navigation. After training on 30 patterns we tested the network and calculated the percent of correctly classified patterns. We applied the same set of patterns to a feed-forward network with one hidden layer. The size of the hidden layer was varied to obtain the best results. The results are summarized in the following table, where each number is averaged over 10 experiments and the standard deviation is also given. 
Table I. Generalization with EKF learning for Connectedness problem Input Size Correctly Classified with CSRN Correctly Classified with MLP

$\begin{array}{ccc}5 \times 5 & 80 \pm 6 \% & 66 \pm 10 \% \\ 6 \times 6 & 82 \pm 6 \% & 65 \pm 12 \% \\ 7 \times 7 & 88.5 \pm 6 \% & 63 \pm 12 \%\end{array}$

We can see that the performance of MLP is just slightly above chance level whereas the CSRN produces correct answers in $80-90 \%$ of test cases on previously unseen patterns. This performance is likely to improve by fine tuning network parameters.

\section{Discussion and Conclusions}

In this contribution we presented the advantages of using the Cellular SRN's as a more general topology compared to conventional MLP. We extended the previous results [27] by applying the CSRN to the connectedness problem. We applied an efficient learning methodology - EKF - to the SRN and obtained very encouraging results in terms of speed of convergence. The unit of our network is a generalized MLP. It can be easily substituted by any other feed-forward computation suitable for problem at hand, without any changes to the Cellular SRN. Now it becomes practical to use the proposed combination of architecture and the training method to any data that has $2 \mathrm{D}$ grid structure. The network size does not grow exponentially with the input size because of the weight sharing. The 100 by 100 input pattern could potentially be processed by the CSRN with 15 units in each cell. However large networks still involve massive computations, which can be possibly addressed by efficient hardware implementations [28].

One example of such application is image processing. Detecting connectedness is a fundamental challenge in this field. We applied our CSRN to a subset of connectedness problem with minimal changes to the code. The results showed that CRSN is much better at recognizing connectedness compared to feed-forward architecture.

Another example of such data is the board games. The games of chess and checkers 
have long been used as testing problems for AI. Recently, neural networks coupled with evolutionary training methods have been successfully applied to the checkers [29] and chess [30]. The neural network architecture used in those works is the case of Object Net [12], mentioned in the introduction. The input pattern (the chess board) is divided into spacial components and the network is built with separate sub-units receiving input from their corresponding components. The interconnections between the sub-units of the network encode the spacial relationships between different parts of the board. The outputs of the Object Net feed into another multilayered network using to evaluate the overall "fitness" of the current situation on the board.

From the above description we can see that the CSRN network is a simplified case of the Object Net. The Chess Object Net belongs to the same class of multistage optimization problems, even though it does not presently use recurrent units. The biggest difference however, is the training method. The evolutionary computation has proven to be able to solve the problem, however at high computational cost. The architecture used in this contribution provides an efficient training method for the Object Net type of networks with more biologically plausible training using local derivatives information. The improved efficiency allows the use of SRN's, which are proven to be more powerful in function approximation than the MLP's. Therefore, the Cellular SRN/EKF can be applicable to many interesting problems.

This contribution builds upon several concepts: Recurrent NN's, Simultaneous Recurrent Networks, Cellular NN's, Dynamic Programming, Kalman Filters. After reviewing each of the concepts, we presented a versatile recurrent neural network architecture capable of efficient training based on EKF methodology. We demonstrate this novel application of EKF on the examples of the maze navigation problem and connectedness problem. Detailed study of properties of EKF for CSRN training is in progress.

\section{Acknowledgements}

The authors would like to thank the anonymous reviewers for detailed comments and useful suggestions. 


\section{References}

[1] D. A. White and D. A. Sofge, Eds., Handbook of Intelligent Control Neural, Fuzzy, and Adaptive Approaches. Van Nostrand Reinhold, 1992.

[2] P. J. Werbos and X. Pang, "Generalized maze navigation: Srn critics solve what feedforward or hebbian cannot," Proc. Conf. Systems, Man, Cybernetics, 1996.

[3] G. K. Venayagamoorthy and G. Singhal, "Quantum-inspired evolutionary algorithms and binary particle swarm optimization for training mlp and srn neural networks," Journal of Computational and Theoretical Nanoscience, vol. 2, pp. 1-8, 2005.

[4] A. R. Barron, "Approximation and estimation bounds for artificial neural networks," Mach. Learn., vol. 14, no. 1, pp. 115-133, 1994.

[5] D. Prokhorov, R. Santiago, and D. Wunsch, "Adaptive critic designs," IEEE Trans. on Neural Networks, vol. 8(5), pp. 997-1007, 1997.

[6] X. Pang and P. Werbos, "Neural network design for $\mathrm{j}$ function approximation in dynamic programming," MATH'L MODELING AND SCIENTIFIC COMP., vol. 2, 1996. [Online]. Available: http://www.citebase.org/abstract?id=oai:arXiv.org:adap-org/9806001

[7] G. V. Puskorius and L. A. Feldkamp, "Neurocontrol of nonlinear dynamical systems with kalman filter trained recurrent networks," IEEE Transactions on Neural Networks, vol. 5, no. 2, pp. 279 - 297, Mar 1994.

[8] L. Feldkamp, D. Prokhorov, C. Eagen, and F. Yuan, Nonlinear Modeling: Advanced Black-Box Techniques, 1998, ch. Enhanced multi-stream kalman filter training for recurrent networks., pp. 29-53.

[9] F. Rosenblatt, Principles of Neural Dynamic. New York: Spartan, 1962.

[10] M. L. Minsky and S. A. Papert, Perceptrons. Cambridge, MA: MIT Press, 1969.

[11] — _ Perceptrons (Expanded Edition). Cambridge, MA: MIT Press, 1988.

[12] L. I. Perlovsky and R. Kozma, Eds., Neurodynamics of Cognition and Consciousness. Springer, 2007,. 
[13] R. Ilin, R. Kozma, and P. J. Werbos, "Cellular srn trained by extended kalman filter shows promise for adp," Proc. Int. J. Conf. NN IJCNN06, pp. 506-510, 2006.

[14] P. Werbos, "Backpropagation through time: What it does and how to do it," Proceedings of the IEEE, vol. 78, pp. 1550-1560, October 1990.

[15] — _ "Consistency of hdp applied to a simple reinforcement learning problem," Neural Networks, vol. 3, pp. 179-189, October 1990.

[16] L. Feldkamp and D. Prokhorov, "Phased backpropagation: A hybrid of temporal backpropagation and backpropagation through time," Proceedings of World Congress on Computational Intelligence, Alaska, 1998.

[17] P. J. Werbos, Handbook of Intelligent Control Neural, Fuzzy, and Adaptive Approaches. Van Nostrand Reinhold, 1992, ch. 3.

[18] R. D. Snyder and C. S. Forbes, "Understanding the kalman filter: an object oriented programming perspective," Monash Econometrics and Business Statistics Working Papers, no. 14/99, 1999.

[19] T. W. Anderson, An Introduction to Multivariate Statistical Analysis. John Wiley and Sons, Inc., 1958.

[20] S. Haykin, Ed., Kalman Filtering and Neural networks. John Wiley and Sons, Inc., 2001.

[21] S. Haykin, Neural Networks, A Comprehensive Foundation. Pearson Education, Inc., 1999.

[22] S. Haykin, Ed., Kalman Filtering and Neural Networks. John Wiley @ Sons, Inc, 2001.

[23] X. Hu, D. V. Prokhorov, and D. C. Wunsch, "Time series prediction with a weighted bidirectional multi-stream extended kalman filter," Neurocomputing, vol. 70, no. 13-15, pp. 2392-2399, 2007.

[24] G. V. Puskorius and L. A. Feldkamp, "Avoiding matrix invensions for the decoupled extended kalman filter training algorithm," Proceeding of the World Congress on Neural Networks, Washington DC, pp. I-764-I-769, 1995. 
[25] D. Wunsch, "The cellular simultaneous recurrent network adaptive critic design for the generalized maze problem has a simple closed-form solution," Proc. International Joint Conference on Neural Networks, 2000.

[26] G. Burdet, P. Combe, and O. Parodi, Eds., Neuronal Information Processing: From Biological Data to Modelling and Applications, ser. Series in Mathematical Biology and Medicine. Singapore: World Scientific, 1999, vol. 7, ch. Algorithms for the detection of connectedness and their neural implementation, by P. R. Roelfsema and S. Bohte and H. Spekreijse.

[27] R. Ilin, R. Kozma, and P. J. Werbos, "Efficient learning in cellular simultaneous recurrent neural networks - the case of maze navigation problem," Proceedings of IEEE International Symposium on Approximate Dynamic Programming and Reinforcement Learning, pp. 324-329, 2007.

[28] T. Yang and L. O. Chua, "Implementing back-propagation though-time learning algorithm using cellular neural networks," International Journal of Bifurcation and Chaos, vol. 9, no. 6, pp. 1041-1074, 1999.

[29] D. B. Fogel and K. Chellapilla, "Evolving an expert checkers playing program without using human expertise," IEEE Transactions on Evolutionary Computation, vol. 5, no. 4, pp. 422-428, 2001.

[30] D. B. Fogel, T. J. Hays, S. L. Hahn, and J. Quon, "A self-learning evolutionary chess program," Proceedings of the IEEE, vol. 92, no. 12, pp. 1947-1954, 2004.

\section{Appendix A: Example of Calculating Ordered Deriva- tives}

The following example is an illustration of the principles mentioned in section 2, Consider the network in Fig. 9 A. This network can be decomposed into 4 identical units as shown in Fig. 9B, where each unit is a mapping between its inputs, internal parameters and outputs. This is a case of a simple recurrent cellular network with two cells and two iterations. We unfold the recurrent steps to demonstrate the application of the rule of ordered derivatives. 
Each unit has 3 inputs $x_{1}, x_{3}$, and $x_{3}$, and 3 parameters $a, b$, and $c$. The outputs of each neuron are denoted by $z_{1}, z_{2}$, and $z_{3}$. The first input neuron does not perform any transformation, so $z_{1}=x_{1}$. The second and third neurons use a nonlinear transformation $f$. The forward calculation of an elementary unit is as follows.

$$
\begin{aligned}
& z_{2}=x_{2}+f\left(c x_{1}\right) \\
& z_{3}=x_{3}+f\left(a x_{1}+b z_{2}\right)
\end{aligned}
$$

The order in which different quantities appear in the forward calculation is

$$
x_{1}, x_{2}, x_{3}, c, z_{2}, a, b, z_{3}
$$

We would like to determine the derivatives of $z_{2}$ and $z_{3}$ w.r.t. the inputs and parameters. To do so, we apply the rule of ordered derivatives [14] given by the following formula.

$$
\frac{\partial^{+} T A R G E T}{\partial z_{i}}=\frac{\partial T A R G E T}{\partial z_{i}}+\sum_{j=i+1}^{N} \frac{\partial^{+} T A R G E T}{\partial z_{j}} \frac{\partial z_{j}}{\partial z_{i}}
$$

where TARGET is the variable the derivative of which w.r.t. $z_{i}$ is sought, and the calculation of TARGET involves using $z_{j}$ 's is order of their subscripts. The notation $\partial^{+}$is used for the ordered derivative, which simply means the full derivative, as opposed to simple partial derivative obtained by considering only the final equation involving TARGET.

In order to calculate the derivatives in our example, we have to use the equation A-4 in reverse order of $\mathrm{A}-3$, Let $\phi$ denote the derivative of $f$.

$$
\begin{aligned}
\frac{\partial^{+} z_{3}}{\partial b} & =\frac{\partial z_{3}}{\partial b}=z_{2} \phi\left(a x_{1}+b z_{2}\right) \\
\frac{\partial^{+} z_{3}}{\partial a} & =\frac{\partial z_{3}}{\partial a}=x_{1} \phi\left(a x_{1}+b z_{2}\right) \\
\frac{\partial^{+} z_{3}}{\partial z_{2}} & =\frac{\partial z_{3}}{\partial z_{2}}=b \phi\left(a x_{1}+b z_{2}\right) \\
\frac{\partial^{+} z_{3}}{\partial c} & =\frac{\partial^{+} z_{3}}{\partial z_{2}} \frac{\partial^{+} z_{2}}{\partial c}=b \phi\left(a x_{1}+b z_{2}\right) x_{1} \phi\left(c x_{1}\right) \\
\frac{\partial^{+} z_{3}}{\partial x_{3}} & =\frac{\partial z_{3}}{\partial x_{3}}=1 \\
\frac{\partial^{+} z_{3}}{\partial x_{2}} & =\frac{\partial^{+} z_{3}}{\partial z_{2}} \frac{\partial z_{2}}{\partial x_{2}}=b \phi\left(a x_{1}+b z_{2}\right) \\
\frac{\partial^{+} z_{3}}{\partial x_{1}} & =\frac{\partial^{+} z_{3}}{\partial z_{2}} \frac{\partial z_{2}}{\partial x_{1}}=b \phi\left(a x_{1}+b z_{2}\right) c \phi\left(c x_{1}\right)
\end{aligned}
$$




$$
\begin{aligned}
\frac{\partial^{+} z_{2}}{\partial c} & =\frac{\partial z_{2}}{\partial c}=x_{1} \phi\left(c x_{1}\right) \\
\frac{\partial^{+} z_{2}}{\partial x_{3}} & =\frac{\partial z_{2}}{\partial x_{3}}=0 \\
\frac{\partial^{+} z_{2}}{\partial x_{2}} & =\frac{\partial z_{2}}{\partial x_{2}}=0 \\
\frac{\partial^{+} z_{2}}{\partial x_{1}} & =\frac{\partial z_{2}}{\partial x_{1}}=c \phi\left(c x_{1}\right)
\end{aligned}
$$

Knowing these derivatives, what are the derivatives of the full network? Let's add the superscript to each variable indicating which unit of the network it belongs to. Note that the outputs of the earlier unit become the inputs of the later unit. Consider unit 2, which gets input from units 1 and 3. If we apply $\mathrm{A}-4$ to obtain the derivative of, for example, $z_{3}^{2}$ w.r.t. $a$, we will get, based on the topology of connections between the units, the following result.

$$
\frac{\partial^{+} z_{3}^{2}}{\partial a}=\frac{\partial^{+} z_{3}^{2}}{\partial a^{2}}+\frac{\partial^{+} z_{3}^{2}}{\partial z_{3}^{3}} \frac{\partial^{+} z_{3}^{3}}{\partial a^{3}}+\frac{\partial^{+} z_{3}^{2}}{\partial z_{2}^{1}} \frac{\partial^{+} z_{2}^{1}}{\partial a^{2}}
$$

Obviously $a^{2}=a^{3}=a$ as we use identical units. The quantities $\frac{\partial^{+} z_{2}^{1}}{\partial a^{2}}$ and $\frac{\partial^{+} z_{3}^{3}}{\partial a^{3}}$ are already obtained for each unit. Since $z_{2}^{1}=x_{3}^{2}$ and $z_{3}^{3}=x_{2}^{2}$, the quantities $\frac{\partial^{+} z_{3}^{2}}{\partial z_{2}^{1}}$ and $\frac{\partial^{+} z_{3}^{2}}{\partial z_{3}^{3}}$ are equivalent to $\frac{\partial^{+} z_{3}^{2}}{\partial x_{3}^{2}}$ and $\frac{\partial^{+} z_{3}^{2}}{\partial x_{2}^{2}}$, which are also already calculated for each individual unit. They are the input "deltas", or the output derivatives "propagated" through the unit backwards. In other words, when all the quantities of each individual unit are calculated, then the total derivatives of the outputs of the full network w.r.t. any parameter are obtained by summing the individual unit's derivative multiplied by the corresponding "delta". The correspondence is determined by the topology of connections - knowing which output is connected to which input. Every time we backpropagate through a unit, we also set the values of the "deltas" of preceding units. In our example:

$$
\begin{aligned}
\delta_{2}^{2} & =\frac{\partial^{+} z_{3}^{2}}{\partial z_{2}^{1}}=\frac{\partial^{+} z_{3}^{2}}{\partial x_{3}^{2}} \\
\delta_{3}^{2} & =\frac{\partial^{+} z_{3}^{2}}{\partial z_{3}^{3}}=\frac{\partial^{+} z_{3}^{2}}{\partial x_{2}^{2}}
\end{aligned}
$$

And 


$$
\frac{\partial^{+} z_{3}^{2}}{\partial a}=\frac{\partial^{+} z_{3}^{2}}{\partial a^{2}}+\delta_{3}^{2} \frac{\partial^{+} z_{3}^{3}}{\partial a^{3}}+\delta_{2}^{2} \frac{\partial^{+} z_{2}^{1}}{\partial a^{2}}
$$

Likewise, in general case, the final calculation for any network output $j$ is a simple summation given by Eq. 1. 


\section{Figure Captions}

- Figure 1; Algorithm for calculating ordered derivatives in recurrent neural network. See section 2 for explanations.

- Figure 2: Generic Cellular SRN architecture.

- Figure 3. Cell of CSRN is a generalized MLP with $\mathrm{m}$ inputs and $\mathrm{n}$ outputs. The solid lines are adjustable weights and the dashed lines are unit weights. Note that the output of the cell is scaled by the output weight.

- Figure 4. Example of 5 by 5 maze world. Black squares are obstacles. $\mathrm{X}$ is the location of the goal. The agent needs to find the shortest path from any white square to the goal.

- Figure 5. Comparison of the solution given by the network and the true solution. A approximate solution, black arrows point in the wrong direction. B - exact solution.

- Figure 6: Examples of input patterns for Connectedness problem for 7 by 7 image.

- Figure 7f A. Average sum squared error for training on 30 mazes and testing on 10. Solid - EKF training error, dotted - EKF testing error, dashed - ALR training error, dash-dot - ALR testing error. The 12.25 threshold for sum squared error is shown by solid line. B. Average Goodness of Navigation G for training on 30 mazes and testing on 10. Solid - EKF training, dotted - EKF testing, dashed - ALR training, dash-dot - ALR testing. The 50 percent solid line is the chance level network.

- Figure 8: Pseudo-code for the training cycle of the CSRN.

- Figure 9: Simple feedforward network (A) which can be divided into 4 blocks (B). 


\section{$11 \quad$ Figures}

Calculation of derivatives in recurrent network

\begin{tabular}{cl}
$\delta_{k}^{N}=1, k=1 . . n$ & Initialize deltas \\
$F_{\alpha}^{k}=\partial z_{k}^{N} / \partial \alpha, k=1 . . n$ & Initialize ordered derivatives \\
For t=N-1 down to 1 & for each time step \\
For $\mathrm{k}=1$ to $\mathrm{n}$ & for each network output \\
$\delta_{k}^{t}=\sum_{p \in \operatorname{fan}-i n(k)} \partial z_{k}^{t+1} / \partial x_{p}^{t}$ & find deltas \\
$F_{\alpha}^{k}=F_{\alpha}^{k}+\delta_{k}^{t} \partial z_{k}^{t} / \partial \alpha$ & update derivatives \\
\hline
\end{tabular}

Figure 1: 


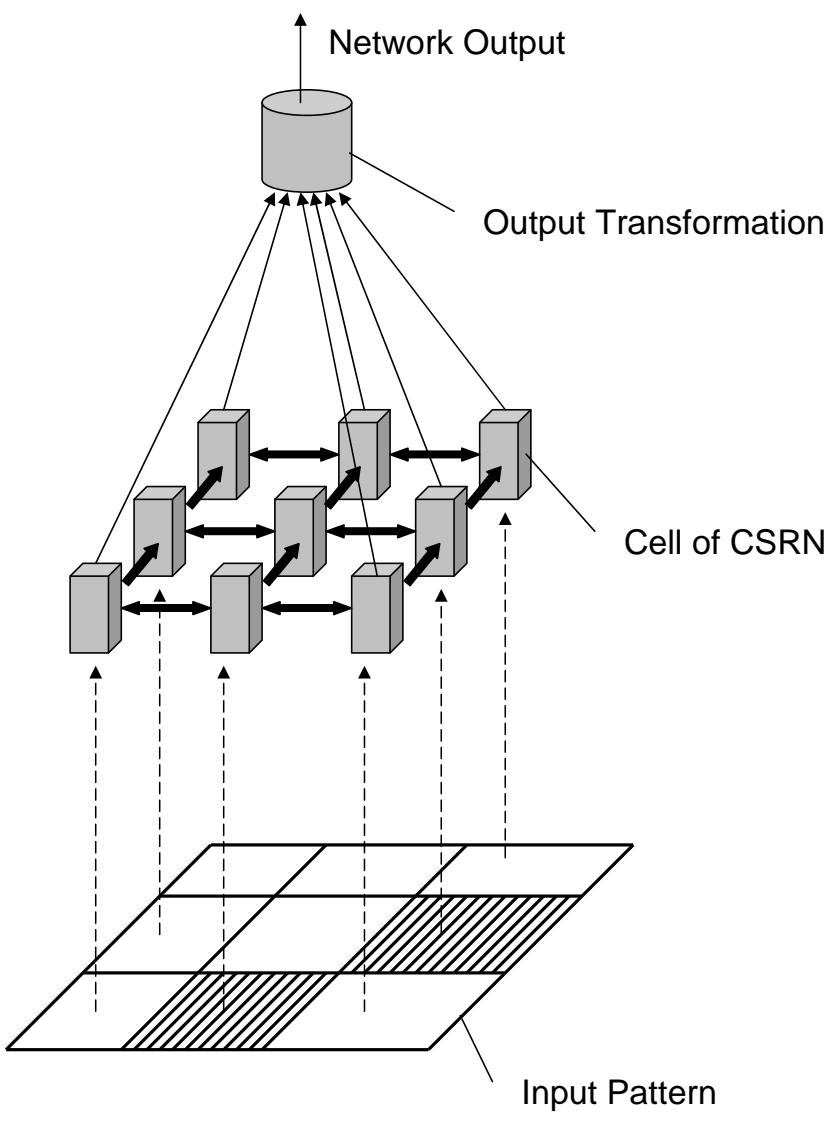

Figure 2: 


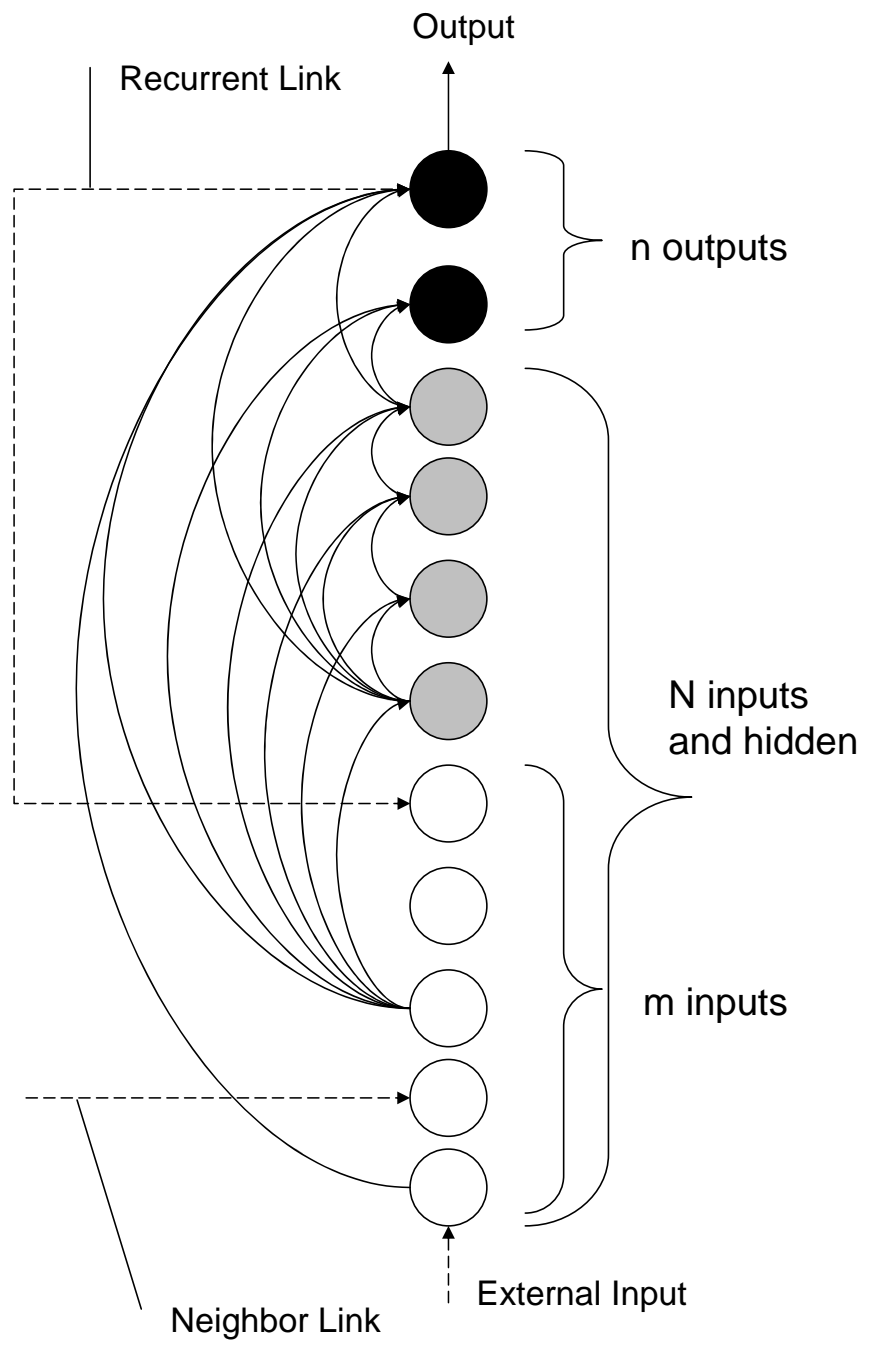

Figure 3: 


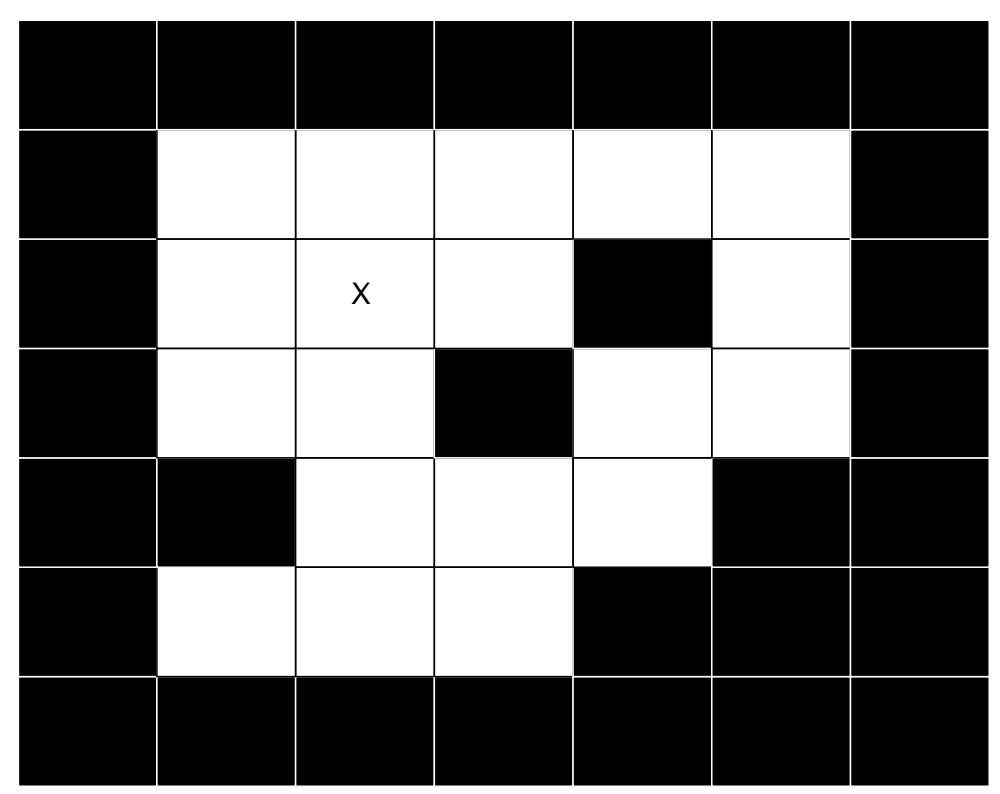

Figure 4: 


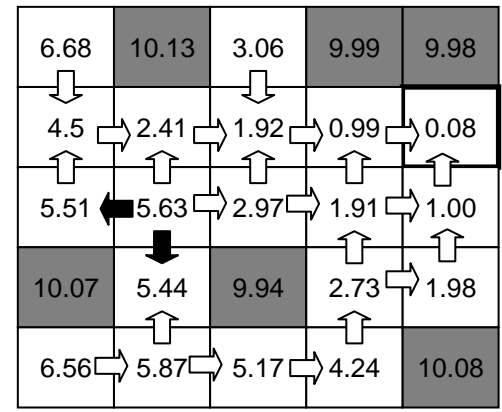

A

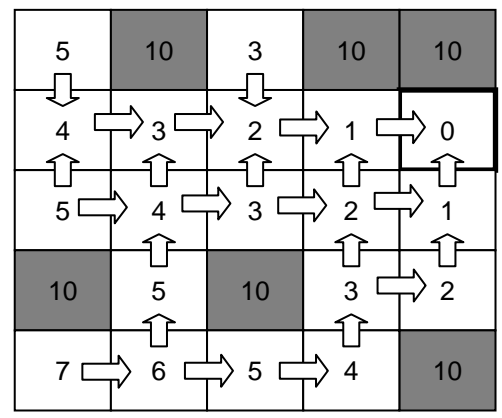

B

Figure 5: 


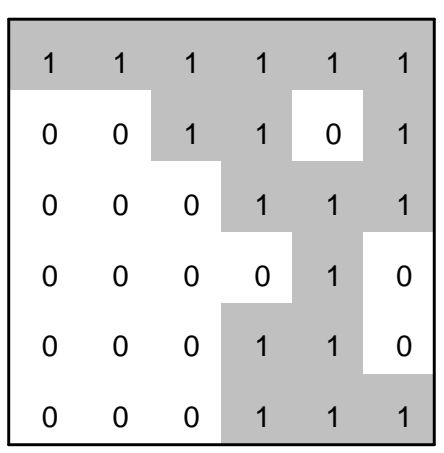

Connected

\begin{tabular}{|llllll|}
\hline 1 & 0 & 0 & 0 & 0 & 1 \\
0 & 0 & 0 & 1 & 1 & 0 \\
0 & 0 & 0 & 1 & 0 & 0 \\
1 & 1 & 1 & 0 & 1 & 0 \\
0 & 0 & 1 & 1 & 1 & 0 \\
1 & 1 & 1 & 0 & 0 & 1 \\
\hline
\end{tabular}

Not connected

Figure 6: 


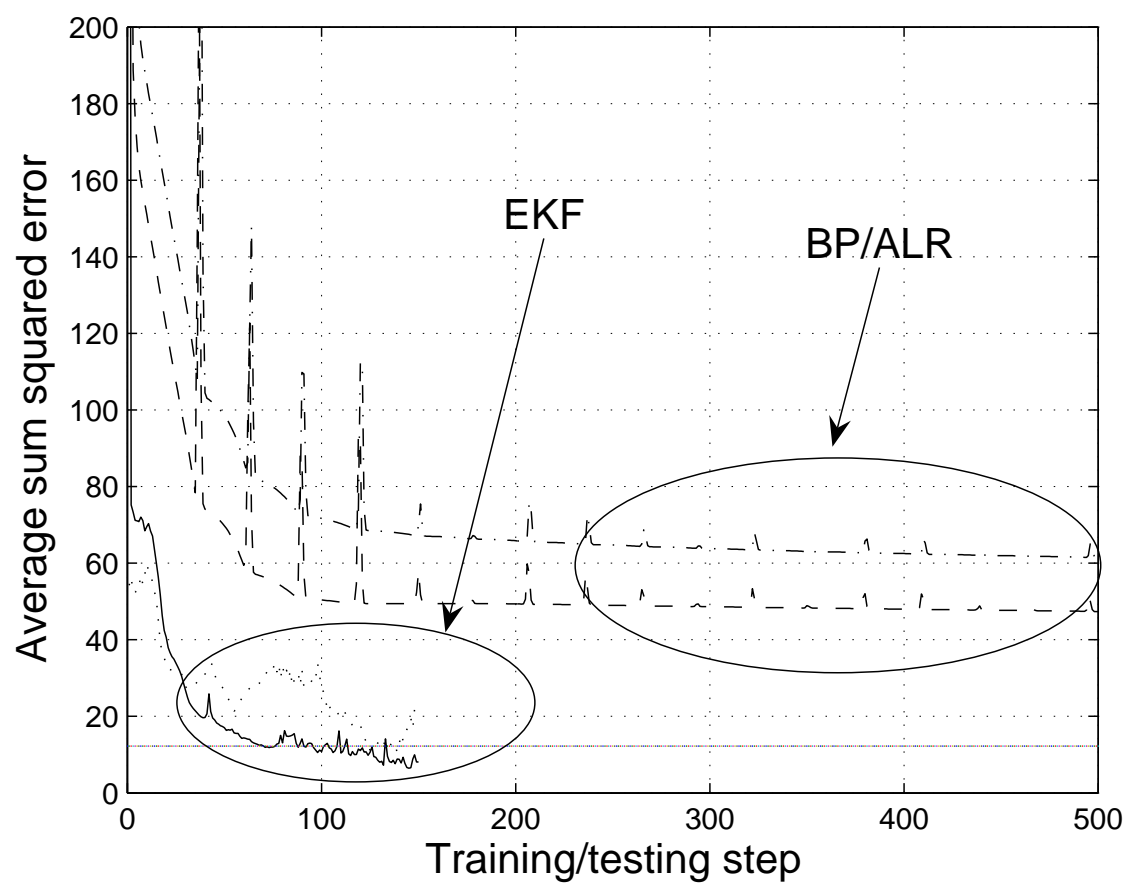

A

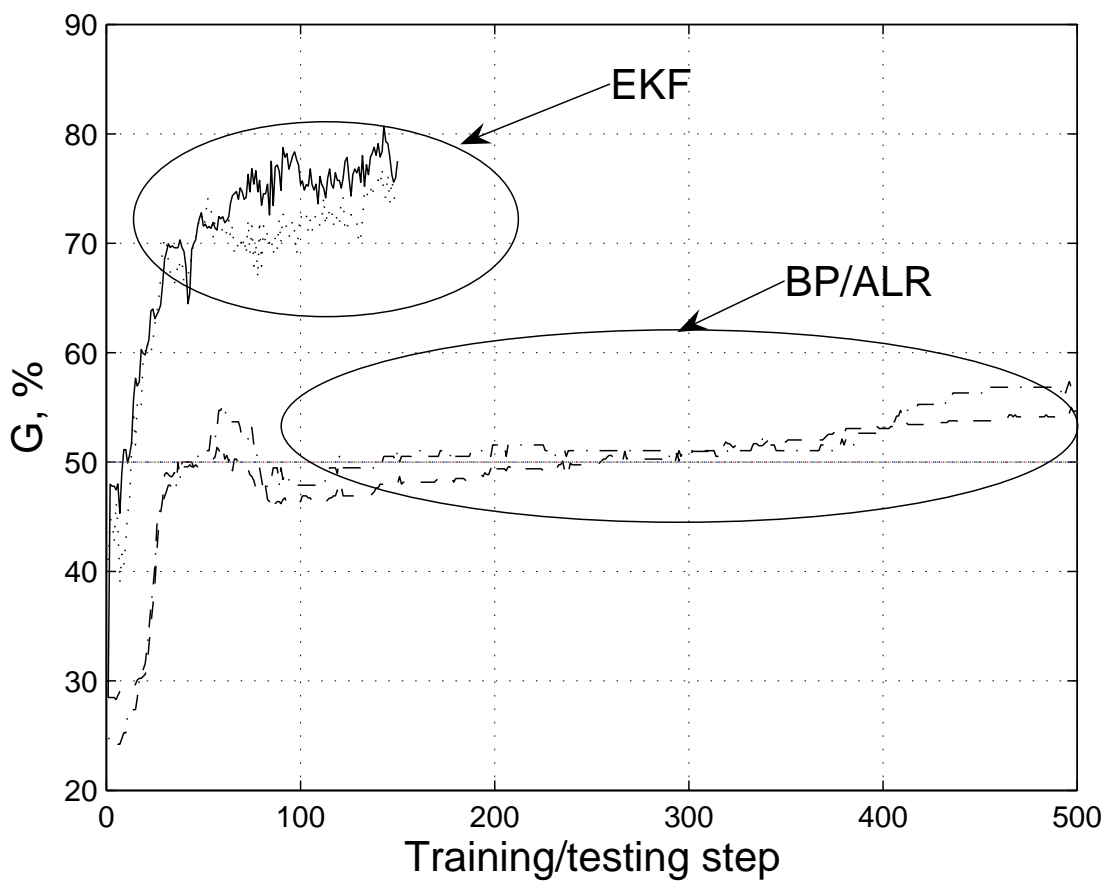

B

Figure 7: 
Training Cycle of CSRN with EKF weight update

Initialize network weights

Initialize EKF parameters Q, R, K

Repeat Until Training is Completed

Set Jacobian C to empty matrix

For each Training Pattern

Run forward update of CSRN

Calculate Network Output(s) and Error

Back propagate Error though Output Transformation

Backpropagate Deltas from Output Transformation through CSRN

Augment the Jacobian matrix C

Calculate weight adjustments using EKF algorithm

For each Testing Pattern (Stopping Criteria)

Run forward update CSRN

Calculate Network Output

Determine whether training is completed

Figure 8: 

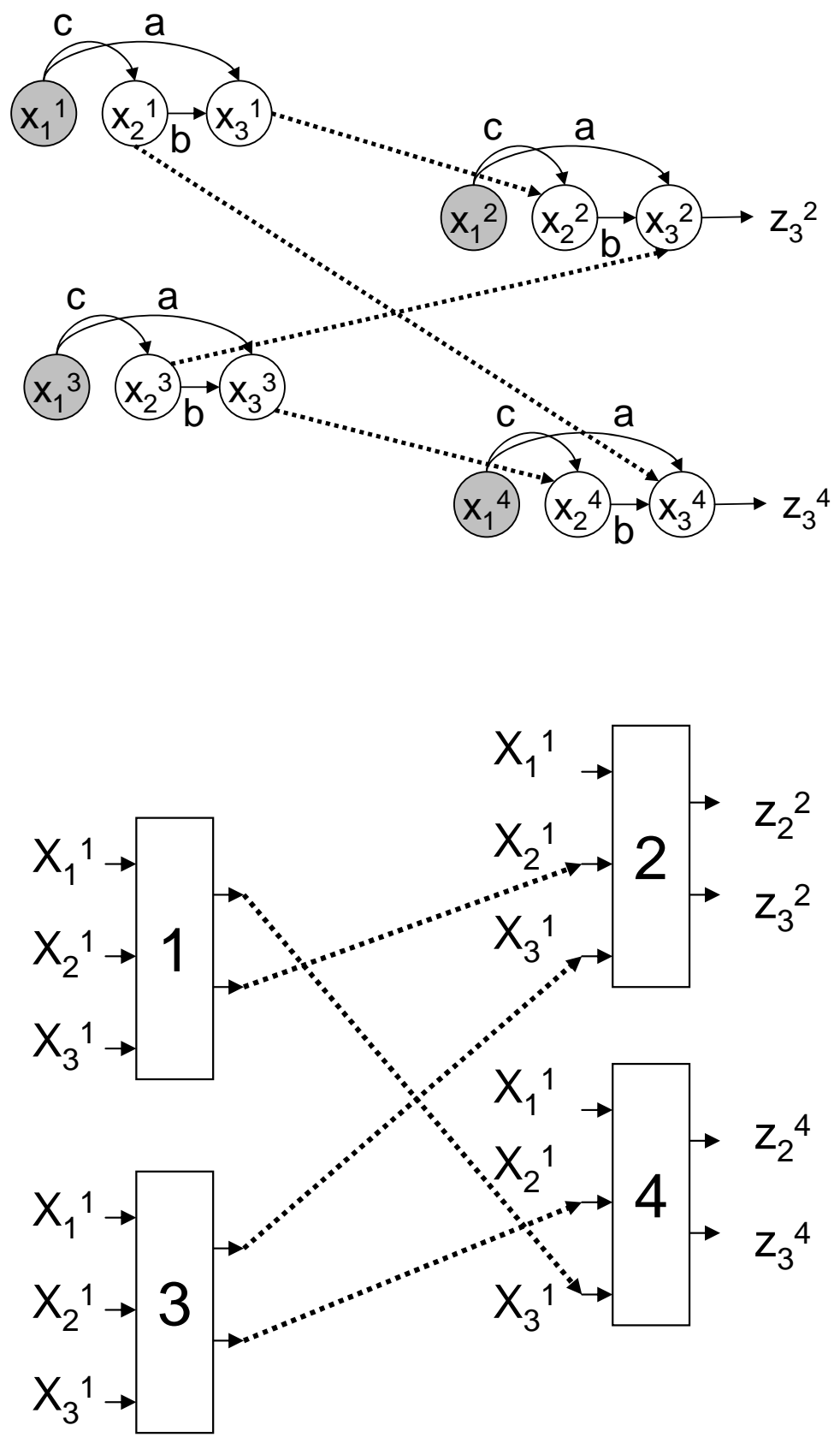

B

Figure 9: 